\title{
Deep bed filtration with multiple pore-blocking mechanisms
}

\author{
Liudmila Kuzmina ${ }^{1}$, and Yuri Osipov ${ }^{2, *}$ \\ ${ }^{1}$ National Research University Higher School of Economics, Myasnitskaya st., 20, Moscow, 101000 \\ Russia \\ ${ }^{2}$ Moscow State University of Civil Engineering, Yaroslavskoe shosse, 26, Moscow, 129337, Russia
}

\begin{abstract}
A one-dimensional model for the deep bed filtration of a monodisperse suspension in a porous medium with variable porosity and permeability and multiple pore-blocking mechanisms is considered. It is assumed that the small pores are clogged by separate particles; pores of medium size, exceeding the diameter of the particles, can be blocked by arched bridges, forming stable structures at the pore throats. These poreblocking mechanisms - size-exclusion and different types of bridging act simultaneously. Exact solutions are obtained for constant coefficients, on the concentrations front and at the porous medium inlet.
\end{abstract}

\section{Introduction}

The filtration problem is one of the main parts of underground hydromechanics. The study of transport and deposition of solid particles in a porous medium is vital for strengthening loose soil, creating watertight walls for underground hazardous waste storage facilities, construction of tunnels and hydraulic structures [1-3].

Deep bed filtration of the fluid flow with tiny solid particles results in the particle deposition and the pore blocking by the retained particles in the entire porous medium. There are many factors that influence the formation of deposit: diffusion, electrostatic and hydrodynamic forces, viscosity, van der Waals forces, and others [4, 5]. If particle and pore size distributions overlap, the main mechanism for particles capture is size-exclusion: particles freely pass through large pores and get stuck at the throats of small pores with sizes smaller than the particle diameter [6]. Experiments show that medium pores, whose dimensions exceed particle sizes, can also be blocked. At a sufficiently high fluid flow rate, the hydrodynamic forces of particle attraction overcome the repulsive electrostatic forces. Suspended particles form groups that lock the pore throats with arched bridges [7, 8].

The model of deep bed filtration of a monodisperse suspension in a homogeneous porous medium with pores of various sizes is considered. Assume that small pores can be blocked by separate particles; the medium-size pores, exceeding the diameter of the particles, can be blocked by arched bridges formed of several particles. All medium pores of the same size, which exceeds the diameter of the particles, may be locked by identical stable bridge constructions. Particles and arched bridges fixed at the pore inlets can not be

\footnotetext{
* Corresponding author: yuri-osipov@mail.ru
} 
knocked out or demolished by a fluid flow or other particles. Large pores, whose dimensions are substantially larger than the particles diameter, can not be blocked, and the carrier fluid with suspended particles flows freely through a porous medium (Fig. 1).

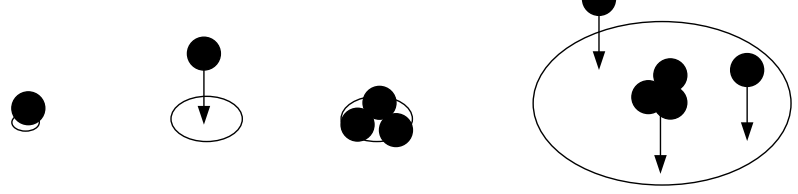

Fig. 1. Particle transport and pore blocking by separate particles and arched bridges.

The basic mathematical model of deep bed filtration includes the mass balance equation for the suspended and retained particles and the kinetic equation of deposit growth [9, 10]. The modified filtration model takes into account the dependence of the porosity and permeability of the porous medium on the retained particles concentration [11]. All these models consider only one mechanism of particle capture. In [12], a model with several particle retention mechanisms is studied. However, these mechanisms are associated with single particles, rather than with composite structures.

The proposed nonlinear model determines the simultaneous action of several geometric pore-blocking mechanisms, including the deposition of individual particles and arched bridges of different configurations. Unlike standard models in which the rate of the deposit growth is proportional to the suspended particles concentration, the growth rate of the deposited arch bridges depends nonlinearly on this concentration.

For some filtration models, exact solutions are obtained [13-15], in the absence of a global exact solution, asymptotics are constructed [16-18], if there are no analytical solutions, numerical methods are used [19, 20].

In this paper, exact solutions are constructed for a one-dimensional filtration problem in a porous medium with varying porosity and permeability and different geometric mechanisms of particle capture acting simultaneously. Global solution of the problem with constant filtration coefficients, local solutions at the concentration front and at the entrance of the porous medium are obtained. Numerical calculation of the solutions is performed.

Section 2 is devoted to the mathematical model of the filtration problem. In Section 3, exact solutions are constructed; the results of numerical calculation are given in Section 4. The Conclusion in Section 5 finalizes the article.

\section{The governing equations}

In the domain

$$
\Omega=\{(x, t): 0<x<1, t>0\}
$$

a one-dimensional filtration problem in a porous medium with variable porosity and permeability and different geometric mechanisms of pore blocking is set by the equations

$$
\begin{gathered}
\frac{\partial}{\partial t}(G(S) C)+\frac{\partial}{\partial x}(F(S) C)+\frac{\partial S}{\partial t}=0 \\
\frac{\partial S_{i}}{\partial t}=\Lambda_{i}\left(S_{i}\right) H_{i}(C), \quad i=1,2, \ldots, n \\
S=\alpha_{1} S_{1}+\alpha_{2} S_{2}+\ldots+\alpha_{n} S_{n}
\end{gathered}
$$


Here $C(x, t) ; S_{i}(x, t)$ are volumetric concentrations of suspended particles and retained arched bridges, porosity $G(S)$, permeability $F(S)$, bridging functions $H_{i}(C)$ and filtration coefficients $\Lambda_{i}(S)$ are smooth positive functions, $\alpha_{1}, \alpha_{2}, \ldots, \alpha_{n}$ - positive constants.

The bridging functions $H_{i}(C)$ are determined by the types of arched bridges. According to the law of reacting masses [21], the bridging functions $H_{i}(C): C^{k_{i}}$, where $k_{i}$ is the amount of particles in the bridge $i$.

The mechanism of size-exclusion blocking of small pores by individual particles is set by the formula $H_{1}(C)=C$.

Assume that a suspension with constant concentration of the suspended particles is injected at the porous medium inlet

$$
\left.C\right|_{x=0}=1
$$

and at the initial moment the porous medium is empty

$$
\left.C\right|_{t=0}=0 ;\left.\quad S_{i}\right|_{t=0}=0, \quad i=1,2, \ldots, n .
$$

The conditions (4), (5) define a unique solution of the system (1) - (3).

The concentrations front $t=\beta x, \beta=G(0) / F(0)$ separating the suspension from the empty porous medium moves with constant velocity $v=F(0) / G(0)$, and divides the domain $\Omega$ into two subdomains

$$
\Omega_{0}=\{(x, t): 0<x<1,0<t<\beta x\} ; \Omega_{S}=\{(x, t): 0<x<1, t>\beta x\} .
$$

In the domain $\Omega_{0}$, the solution is zero; in the domain $\Omega_{S}$ the solution is positive. At the concentrations front, the solution $C(x, t)$ is discontinuous; the solution $S(x, t)$ is continuous and has discontinuous derivatives.

\section{Global and local exact solutions}

\subsection{Solution of the problem with constant coefficients}

Let $G(S)=G, F(S)=F, \Lambda_{i}(S)=\Lambda_{i}, i=1, \ldots, n$. Equations (1), (2) take the form

$$
\begin{gathered}
G \frac{\partial C}{\partial t}+F \frac{\partial C}{\partial x}+\frac{\partial S}{\partial t}=0 \\
\frac{\partial S_{i}}{\partial t}=\Lambda_{i} H_{i}(C), \quad i=1,2, \ldots, n .
\end{gathered}
$$

The substitution of (3), (7) into equation (6) gives

$$
G \frac{\partial C}{\partial t}+F \frac{\partial C}{\partial x}+\sum_{i=1}^{n} \alpha_{i} \Lambda_{i} H_{i}(C)=0
$$


In the domain $\Omega_{S}$, the exact solution of equation (8) with condition (4) is given by the implicit formula

$$
\int_{1}^{C} \frac{d c}{\sum_{i=1}^{n} \alpha_{i} \Lambda_{i} H_{i}(c)}=-\frac{x}{F}
$$

Since the solution (9) does not depend on time, the solution of equations (7) in $\Omega_{S}$ has the form

$$
S_{i}(x, t)=\Lambda_{i} H_{i}(C)(t-\beta x) ; \quad i=1,2, \ldots, n .
$$

\subsection{Solution on the concentrations front}

On the concentrations front $\Gamma$, the concentrations of partial deposits are zero, and equation (1) takes the form (6). Similarly to Sec. 3.1 the concentrations of suspended and retained particles on the front are given by the formulas

$$
\int_{1}^{\left.C(x, t)\right|_{\Gamma}} \frac{d c}{\sum_{i=1}^{n} \alpha_{i} \Lambda_{i}(0) H_{i}(c)}=-\frac{x}{F(0)} ;\left.\quad S_{i}(x, t)\right|_{\Gamma}=0 ; i=1, \ldots, n ;\left.\quad S(x, t)\right|_{\Gamma}=0 .
$$

\subsection{Solution at the porous medium inlet}

According to the condition (3), at the porous medium inlet $x=0$ the kinetic equations for the deposit growth take the form

$$
\frac{\partial S_{i}}{\partial t}=\Lambda_{i}\left(S_{i}\right) H_{i}(1), \quad i=1,2, \ldots, n
$$

The solutions of equations (12) with conditions (5) are given implicitly by the formulas

$$
\int_{0}^{S_{i}(0, t)} \frac{d s}{\Lambda_{i}(s)}=H_{i}(1) t ; \quad i=1, \ldots, n .
$$

\section{Numerical calculation}

Numerical calculations are performed for a basic example

$$
n=2 ; H_{1}=C ; H_{2}=C^{3} \text {. }
$$

Calculation of the solution (9), (10) is carried out for constant coefficients $F=G=1$, $\Lambda_{1}=2, \Lambda_{2}=1$.

Fig. 2 shows the concentration of suspended particles at $t=0.5$ and $t=1$. 


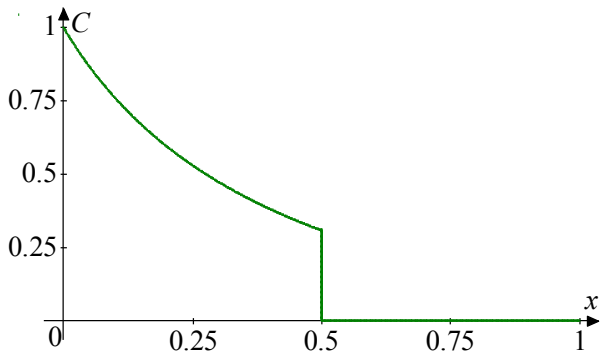

Fig. 2. a) $\left.C(x, t)\right|_{t=0.5}$.

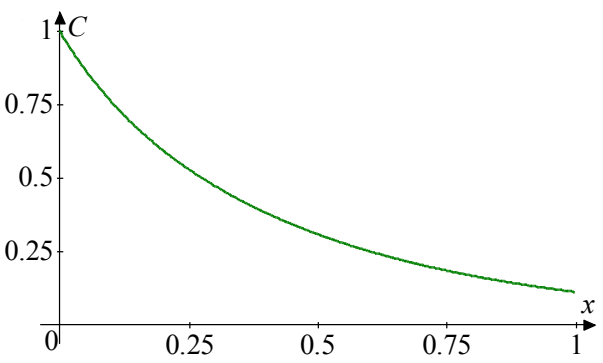

b) $\left.C(x, t)\right|_{t=1}$.

The graphs of the retained particles concentrations ( $S_{1}$ - dashed line, $S_{2}$ - broken line) at $t=0.5, t=1$ are presented in Fig. 3 .

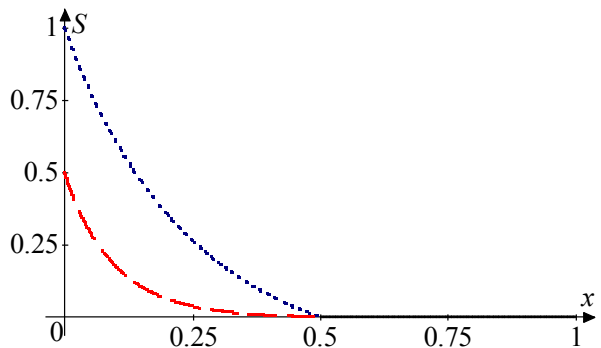

Fig. 3. a) $\left.S_{i}(x, t)\right|_{t=0.5}$.

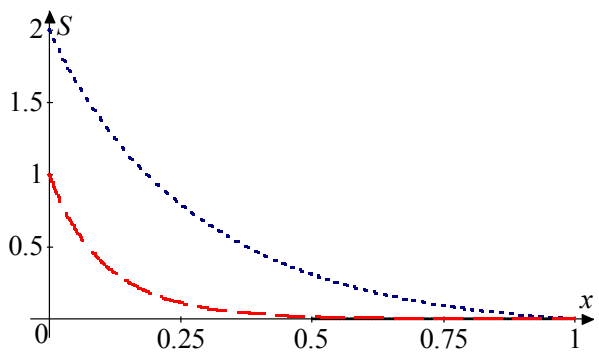

b) $\left.S_{i}(x, t)\right|_{t=1}$.

Fig. 2, 3 show that for $t=0.5$ the solution $C(x, t)$ has a discontinuity on the concentrations front at the point $x=0.5$; the solutions $S_{i}(x, t) ; i=1,2$ are continuous. At $t=1$ the concentrations front reaches the porous medium outlet $x=1$, and all the solutions are continuous.

A solution (13) at the porous medium inlet is obtained for linear filtration coefficients

$$
\Lambda_{1}=2-S_{1} ; \Lambda_{2}=1-S_{2} .
$$

Graphs of the retained particles concentrations ( $S_{1}$ - dashed line, $S_{2}$ - broken line) at $x=0$ are presented in Fig. 4 .

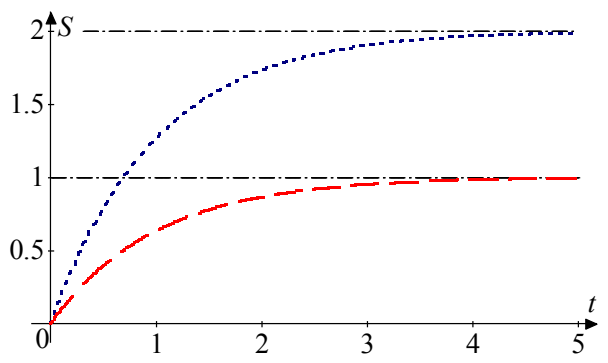

Fig. 4. Retained particles concentrations $\left.S_{i}(x, t)\right|_{x=0}$.

In Fig. 4 partial deposit concentrations $S_{i}(0, t) ; i=1,2$ tend to the maximum limit values with increasing time. These limits are the roots of the filtration coefficients (14). 


\section{Conclusion}

A new nonlinear model of long-term deep bed filtration with variable porosity and permeability and several pore blocking mechanisms is considered, including pore clogging by single particles and arched bridges of different configurations. The properties of solutions are studied. Exact global solutions for constant coefficients and local solutions on the characteristics of a nonlinear hyperbolic system are constructed.

It is shown that the solution has a discontinuity on the concentrations front of the suspended and retained particles. Before the front, the solution is zero, the solution behind the front is positive.

The exact solutions obtained will serve as the basis for constructing local asymptotics in the neighborhood of the characteristics and global asymptotic solution of the problem with almost constant coefficients.

Analytical solutions of the filtration problem determine the dependence of the suspended and retained particles concentrations on external parameters. Variation of the parameters gives way for fine tuning of laboratory experiments [22].

\section{References}

1 Q. Zhang, P. Li, X. Zhang S. Li, W. Zhang, Q. Wang, Open Civ. Eng. J. 9, 1 (2015)

2 M. Tsuji, S. Kobayashi, S. Mikake, T. Sato, H. Matsui, Proc. Eng. 191 (2017)

3 L. Faramarzi, A. Rasti, S.M. Abtahi, Constr. Build. Mat. 126, 32-43 (2016)

4 D. Mays, J.R. Hunt, J. Environ. Sci. Technol. 39, 577-584 (2005)

5 C.V. Chrysikopoulos, V.I. Syngouna, J. Environ. Sci. Technol. 48, 6805-6813 (2014)

6 Z. You, A. Badalyan, P. Bedrikovetsky, SPE J. 18, 620-633 (2013)

7 S. Torkzaban, J. Wan, T.K. Tokunaga, S.A. Bradford, J. Contam. Hydrol. 136-137, 86-95 (2012)

8 V. Ramachandran, H.S. Fogler, J. Fluid Mech. 385, 129-156 (1999)

9 J.P. Herzig, D.M. Leclerc, P. Legoff, Ind. Eng. Chem. 62, 8, 8-35 (1970)

10 F. Civan, Reservoir formation damage (Fundamentals, Modeling, Assessment, and Mitigation) (Gulf Prof. Publ., 2014)

11 A. Santos, P. Bedrikovetsky, S. Fontoura, J. Memb. Sci. 308, 115-127 (2008)

12 R.G. Guedes, F.A.H. Al-Abduwani, P. Bedrikovetsky, P.K. Currie, SPE J. 14, 3 (2009)

13 E.A Vyazmina, P.G. Bedrikovetskii, A.D. Polyanin, Theor. Found. Chem. Eng. 41, 5, 556-564 (2007)

14 S. Borazjani, P. Bedrikovetsky, Appl. Math. Model. 44, 296-320 (2017)

15 N.E. Leont'ev, Fluid Dyn. 52, 1, 165-170 (2017)

16 D. Andreucci, A.F. Tedeev, Comm. Part. Diff. Eq. 42, 3, 347-365 (2017)

17 L.I. Kuzmina, Yu.V. Osipov, Y.P. Galaguz, Int. J. Non-Lin. Mech. 93, 1-6 (2017)

18 Z. You, Y. Osipov, P. Bedrikovetsky, L. Kuzmina, Chem. Eng. J. 258, 374-385 (2014)

19 Y.P. Galaguz, G.L. Safina, MATEC Web Conf. 86, 03003 (2016)

20 Y.P. Galaguz, G.L. Safina, MATEC Web Conf. 117, 00052 (2017)

21 H.S. Fogler, Elements of Chemical Reaction Engineering (Prentice Hall PTR, 2006)

22 A Vaz, P. Bedrikovetsky, P.D. Fernandes, A. Badalyan, T. Carageorgos, J. Petrol. Sci. Eng. 151, 421-433 (2017) 